Supplement of Biogeosciences, 15, 5395-5413, 2018

https://doi.org/10.5194/bg-15-5395-2018-supplement

(C) Author(s) 2018. This work is distributed under

the Creative Commons Attribution 4.0 License.

(c) (1)

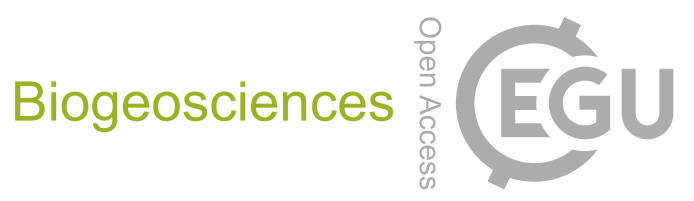

Supplement of

\title{
Synthetic ozone deposition and stomatal uptake at flux tower sites
}

Jason A. Ducker et al.

Correspondence to: Jason A. Ducker (jad10d@my.fsu.edu)

The copyright of individual parts of the supplement might differ from the CC BY 4.0 License. 
Several methods for calculating stomatal conductance and $\mathrm{O}_{3}$ deposition velocity from eddy covariance measurements are found in literature (e.g. Wesely and Hicks, 1977; Gerosa et al., 2005; Fares et al., 2010). While we follow the same general approach, we present the methods here for completeness and to point out some particular choices we have made. These expressions are used in Eqs. 1-3. The required input variables are $\mathrm{O}_{3}$ mole fraction $\left(\mathrm{mol} \mathrm{mol}^{-1}\right)$, temperature $(\mathrm{K})$, pressure $(\mathrm{Pa})$, specific humidity $\left(\mathrm{kg} \mathrm{kg}^{-1}\right)$, friction velocity $\left(\mathrm{m} \mathrm{s}^{-1}\right)$, sensible and latent heat

The aerodynamic and quasi-laminar layer resistances are calculated from measurements of momentum flux using the Monin-Obukhov similarity relations. For heat, $\mathrm{O}_{3}$, and other gases, the aerodynamic resistance $\left(r_{a}, \mathrm{~s} \mathrm{~m}^{-1}\right)$ is (Foken, 2017, pp. 219-223)

$$
r_{a}=\frac{1}{k u_{*}}\left[\ln \left(\frac{z-d}{z_{0}}\right)-\psi_{H}\left(\frac{z-d}{L}\right)+\psi_{H}\left(\frac{z_{0}}{L}\right)\right]
$$

where $r_{a}$ is evaluated at height $z, u_{*}$ is the friction velocity, $z_{0}(\mathrm{~m})$ is the roughness length for momentum, $d(\mathrm{~m})$ is the displacement height, $k=0.4$ is the von Karman constant, $\psi_{H}(\zeta)$ is the stability function for sensible heat discussed below, and $L$ is the Obukhov length $(\mathrm{m})$. The roughness and displacement heights are $z_{0}=0.1 z_{c}$ and $d=0.7 z_{c}$, respectively, where $z_{c}$ is the canopy height specific to each site (http://fluxnet.fluxdata.org/sites/site-summary/, accessed 24 February 2017). Since canopy heights are not specified for croplands or grasslands in this database, we use a constant canopy height of $1 \mathrm{~m}$ for grasslands and typical crop-specific heights for each agricultural site (Weaver and Bruner, 1927). The stability function is (Foken, 2017, pp. 54-62; Högström, 1988)

$$
\psi_{H}(\zeta)=\left\{\begin{array}{cl}
2 \ln \left(\frac{1+0.95(1-11.6 \zeta)^{1 / 2}}{2}\right) & \text { for } \zeta<0 \\
1-\left(1+\frac{2}{3} \zeta\right)^{\frac{3}{2}}-b_{1}\left(\zeta-\frac{b_{2}}{b_{3}}\right) \exp \left(-b_{3} \zeta\right)-\frac{b_{1} b_{2}}{b_{3}} & \text { for } \zeta \geq 0
\end{array}\right.
$$

where $b_{1}=0.667, b_{2}=5$, and $b_{3}=0.35$. The form above is appropriate for strongly stable conditions $((z-d) / L=\zeta>1)$, which occur frequently in the FLUXNET2015 data, as well weak stability (Beljaars and Holtslag, 1991).

The Obukhov length is (Foken, 2017, pp. 54-62)

$$
L=-\frac{u_{*}^{3} \theta_{v}}{k g\left(\overline{w^{\prime} \theta_{v}^{\prime}}\right)}
$$

where $\theta_{v}$ is virtual potential temperature, $\overline{w^{\prime} \theta_{v}^{\prime}}$ is the vertical flux of virtual potential temperature or buoyancy at the surface, and $g$ is acceleration due to gravity. For calculations, we expand $\theta_{v}$ and $\overline{w^{\prime} \theta_{v}^{\prime}}$ in terms of measured quantities so 


$$
L=-\frac{u_{*}^{3} c_{p} \rho \theta(1+0.61 q)}{k g\left(H(1+0.61 q)+0.61 c_{p} \theta E\right)}
$$

35 where $c_{p}$ is specific heat capacity of air $\left(\mathrm{J} \mathrm{kg}^{-1} \mathrm{~K}^{-1}\right), \rho$ is the mass density of air $\left(\mathrm{kg} \mathrm{m}^{-3}\right), \theta$ is potential temperature $(\mathrm{K}), q$ is specific humidity $\left(\mathrm{kg} \mathrm{kg}^{-1}\right), H$ is the surface sensible heat flux (W $\mathrm{m}^{-2}$ ), and $E$ is the surface moisture flux $\left(\mathrm{kg} \mathrm{m}^{-2} \mathrm{~s}^{-1}\right) . H$ and $E$ are defined positive for upward fluxes.

The quasi-laminar layer resistance for $\mathrm{O}_{3}$ and $\mathrm{H}_{2} \mathrm{O}$ is (Foken, 2017, pp. 219-223)

$$
r_{b}=\frac{2}{k u_{*}}\left(\frac{\mathrm{Sc}}{\mathrm{Pr}}\right)^{2 / 3}
$$

where $\mathrm{Sc}=v / D$ is the Schmidt number, which is the ratio of kinematic viscosity of air $(v)$ to the molecular diffusivity of the gas in air $(D)$, and $\operatorname{Pr}=v / D_{H}$ is the Prandtl number, which involves the thermal diffusivity $\left(D_{H}\right)$. The conductance for heat is the same as Eq. A5, but uses the thermal diffusivity of air in place of molecular diffusivity.

We calculate stomatal resistance and conductance from the evaporative-resistance form of the Penman-Monteith equation (Monteith, 1981; Gerosa et al., 2007). For water vapor,

$$
g_{s, w}^{-1}=r_{s, w}=\frac{\varepsilon \rho\left(e_{s}\left(T_{f}\right)-e\right)}{p E}-\left(r_{a}+r_{b, w}\right)
$$

where $\varepsilon=0.622$ is the mass ratio of $\mathrm{H}_{2} \mathrm{O}$ and dry air, $p$ is the air pressure, $e_{s}\left(T_{f}\right)$ is the saturation vapor pressure at the transpiring leaf surface with temperature $T_{f}, e$ is vapor pressure at the flux measurement height, and $r_{b, w}$ is the quasi-laminar layer resistance to water vapor (Eq. A5). Leaf temperature is not a standard FLUXNET2015 variable, but it can be estimated from sensible heat flux using surface energy balance (Gerosa et al., 2007):

$$
T_{f}=T+\frac{H}{c_{p} \rho}\left(r_{a}+r_{b, H}\right)
$$

where $T$ is the air temperature at the measurement height and $r_{b, H}$ is the quasi-laminar layer resistance to heat (Eq. A5). We initially inverted Monteith's (1981) original equation for evapotranspiration (Eq. 4 in Gerosa et al., 2007) in place of Eq. A6, but the resulting $g_{s, w}$ estimates were much more noisy. Although the forms are analytically equivalent (Gerosa et al., 2007), inverting the evaporative-resistance form is numerically preferable because it avoids subtractive terms that amplify relative errors and it more accurately treats temperature and pressure effects, particularly the non-linearity in the saturation vapor pressure.

The stomatal conductance of $\mathrm{O}_{3}$ is less than water vapor due to its greater molar mass and 66 diffusion against the net gas flow out of the stomatal pore (Marrero and Mason, 1972), so

$$
g_{s}=0.6 g_{s, w} \text {. }
$$


67 In all equations, we include the temperature and pressure dependences of $\rho, c_{p}, v, D, D_{H}$, and

68 latent heat of vaporization and also the humidity dependence of $\rho, c_{p}$, and $D_{H}$ using expressions

69 from Jacobson (2005).

\section{S2 Propagation of uncertainty}

We estimate uncertainties in all derived quantities using standard techniques for propagation of errors (e.g. Taylor, 1997, pp. 73-77). In the following section, $f$ is a function that depends on variables $x_{1}, x_{2}, \ldots x_{n}$ that each have uncertainties $\sigma_{x_{1}}, \sigma_{x_{2}}, \ldots \sigma_{x_{n}}$. The standard error $\left(\sigma_{f}\right)$ in $f\left(x_{1}, x_{2}, \ldots x_{n}\right)$ at time $i$ is approximately

$$
\sigma_{f, i}^{2}=\sum_{j=1}^{n}\left(\frac{\partial f_{i}}{\partial x_{j}}\right)^{2} \sigma_{x_{j}, i}^{2} .
$$

This form neglects covariance between the measurement errors, which is unknown in our case, and is most accurate when $\sigma_{x_{j}} \ll x_{j}$. We use centered finite differences to calculate numerical derivatives through all equations.

The propagation of errors reveals that $F_{\mathrm{s}, \mathrm{O}_{3}}^{\mathrm{syn}}$ and other quantities have errors or uncertainties that vary widely from hour to hour. Daily and monthly averages should account for the varying confidence in each value in the average (e.g. Taylor, 1997, pp. 173-177). For values $f_{i}$ that are from a single distribution, but have different uncertainties $\sigma_{f, i}$, the maximum likelihood estimate of $f$ is

$$
\bar{f}=\left(\sum_{i=1}^{m} w_{i} f_{i}\right)\left(\sum_{i=1}^{m} w_{i}\right)^{-1} ; \quad w_{i}=\sigma_{f, i}{ }^{-2} .
$$

The weights $w_{i}$ reflect the confidence in value $f_{i}$ and the summation is carried out over all times $m$ within the desired averaging period. The standard error of $\bar{f}$ is

$$
\sigma_{\bar{f}}=\left(\sum_{i=1}^{m} w_{i}\right)^{-\frac{1}{2}}
$$

For averaging across times when $f$ is expected to change, as during different hours of the day, an unweighted average is more appropriate

$$
\bar{f}=\frac{1}{m} \sum_{i=1}^{m} f_{i}
$$

95 and the standard error of $\bar{f}$, given by Eq. B1, simplifies to

$$
\sigma_{\bar{f}}=\left(\frac{1}{m^{2}} \sum_{i=1}^{m} \sigma_{f, i}^{2}\right)^{\frac{1}{2}} .
$$


$97 \quad \mathbf{S 3}$ Stomatal and non-stomatal $\mathbf{O}_{3}$ deposition at Harvard Forest

Our estimate of the non-stomatal fraction of $\mathrm{O}_{3}$ deposition at Harvard Forest (mean $8 \%$, range 33 to $34 \%$; Sect. 3.2) is smaller than was previously reported at that site (mean $40 \%$, range 20 $60 \%$; Clifton et al., 2017). The main reason for the different results is the re-calibration of the water vapor fluxes in this work, which is described in Sect. 2.2. Here, we show how other differences between our analysis and that of Clifton et al. (2017) affect the estimate of nonstomatal fraction of $\mathrm{O}_{3}$ deposition at Harvard Forest. Using our gap-filled data, the mean estimate of the non-stomatal fraction of $\mathrm{O}_{3}$ deposition at Harvard Forest does not change but the range slightly increases ( $8 \%$, range -36 to $38 \%$ ). With uncorrected water vapor fluxes, our estimate would be $51 \%$ (range: $32 \%$ to $63 \%$ ). If we also ignore the propagated uncertainty, which varies from hour to hour, and calculate averages with equal weight (i.e. equal uncertainty)

109 for each time interval, as Clifton et al. did, then we would estimate 53\% (range: $34 \%$ to $66 \%$ ). If we also use data filtering criteria from Clifton et al. (i.e. remove $3 \sigma$ outliers of $v_{d}$ and $g_{s}$, but no filtering for precipitation and high relative humidity), then we would estimate $48 \%$ (range: $28 \%$ to $61 \%$ ). Finally, if we also restrict our averages to $9 \mathrm{am}-3 \mathrm{pm}$, as Clifton et al. did, instead of all 113 daylight data, then we would estimate $45 \%$ (range: $25 \%$ to $60 \%$ ). This final estimate is very 114 close to the method and value reported by Clifton et al. (2017). The remaining small differences are probably due to Clifton et al. including 1992 in their analysis and differences in the form of the Penman-Monteith and stability functions. Since the re-calibration of water vapor fluxes (Sect. 2.2) is an improvement in this work and the main reason for our results differing from Clifton et 120 al. (2017), our estimates of small non-stomatal fraction $\mathrm{O}_{3}$ deposition at Harvard Forest appear to be most reliable estimate for this site. 
121 Table S1. Description of FLUXNET2015 Tier 1 sites used in SynFlux.

\begin{tabular}{|c|c|c|c|c|c|c|}
\hline Site name & $\mathrm{PFT}^{1}$ & $\mathrm{Lat}^{2}$ & Lon $^{3}$ & $\mathrm{Clim}^{4}$ & Period & References $^{5}$ \\
\hline AT-Neu & GRA & 47.1167 & 11.3175 & Unk & $2002-2012$ & (Wohlfahrt et al., 2008) \\
\hline BE-Bra & $\mathrm{MF}$ & 51.3092 & 4.5206 & Unk & $1996-2014$ & (Carrara et al., 2004) \\
\hline BE-Lon & $\mathrm{CRO}$ & 50.5516 & 4.7461 & $\mathrm{Cfb}$ & $2004-2014$ & (Moureaux et al., 2006) \\
\hline BE-Vie & $\mathrm{MF}$ & 50.3051 & 5.9981 & $\mathrm{Cfb}$ & $1996-2014$ & (Aubinet et al., 2001) \\
\hline $\mathrm{CH}-\mathrm{Cha}$ & GRA & 47.2102 & 8.4104 & Unk & $2005-2014$ & (Merbold et al., 2014) \\
\hline CH-Dav & ENF & 46.8153 & 9.8559 & Unk & $1997-2014$ & (Zielis et al., 2014) \\
\hline CH-Fru & GRA & 47.1158 & 8.5378 & Unk & $2005-2014$ & (Imer et al., 2013) \\
\hline CH-Lae & $\mathrm{MF}$ & 47.4781 & 8.3650 & Unk & 2004-2014 & (Etzold et al., 2011) \\
\hline $\mathrm{CH}-\mathrm{Oe} 1$ & GRA & 47.2858 & 7.7319 & Unk & $2002-2008$ & (Ammann et al., 2009) \\
\hline $\mathrm{CH}-\mathrm{Oe} 2$ & $\mathrm{CRO}$ & 47.2863 & 7.7343 & Unk & 2004-2014 & (Dietiker et al., 2010) \\
\hline CZ-BK1 & ENF & 49.5021 & 18.5369 & Unk & 2004-2008 & (Acosta et al., 2013) \\
\hline CZ-BK2 & GRA & 49.4944 & 18.5429 & Unk & 2004-2006 & - \\
\hline CZ-wet & WET & 49.0247 & 14.7704 & Unk & $2006-2014$ & (Dûsek et al., 2012) \\
\hline DE-Akm & WET & 53.8662 & 13.6834 & $\mathrm{Cfb}$ & 2009-2014 & - \\
\hline DE-Geb & $\mathrm{CRO}$ & 51.1001 & 10.9143 & Unk & $2001-2014$ & (Anthoni et al., 2004) \\
\hline DE-Gri & GRA & 50.9500 & 13.5126 & $\mathrm{Cfb}$ & 2004-2014 & (Prescher et al., 2010a) \\
\hline DE-Hai & $\mathrm{DBF}$ & 51.0792 & 10.4530 & Unk & $2000-2012$ & (Knohl et al., 2003) \\
\hline DE-Kli & $\mathrm{CRO}$ & 50.8931 & 13.5224 & $\mathrm{Cfb}$ & 2004-2014 & (Prescher et al., 2010) \\
\hline DE-Lkb & ENF & 49.0996 & 13.3047 & Unk & 2009-2013 & (Lindauer et al., 2014) \\
\hline DE-Obe & ENF & 50.7867 & 13.7213 & $\mathrm{Cfb}$ & $2008-2014$ & - \\
\hline DE-RuR ${ }^{6}$ & GRA & 50.6219 & 6.3041 & Unk & $2011-2014$ & (Post et al., 2015) \\
\hline DE-RuS ${ }^{6}$ & $\mathrm{CRO}$ & 50.8659 & 6.4472 & $\mathrm{Cfb}$ & $2011-2014$ & (Mauder et al., 2013) \\
\hline DE-Seh & $\mathrm{CRO}$ & 50.8706 & 6.4497 & Unk & $2007-2010$ & (Schmidt et al., 2012) \\
\hline DE-SfN & WET & 47.8064 & 11.3275 & Unk & $2012-2014$ & (Hommeltenberg et al., 2014) \\
\hline DE-Spw & WET & 51.8923 & 14.0337 & $\mathrm{Cfb}$ & $2010-2014$ & - \\
\hline DE-Tha & ENF & 50.9624 & 13.5652 & $\mathrm{Cfb}$ & 1996-2014 & (Grünwald and Bernhofer, 2007) \\
\hline DK-Fou & $\mathrm{CRO}$ & 56.4842 & 9.5872 & Unk & $2005-2005$ & - \\
\hline DK-Sor & $\mathrm{DBF}$ & 55.4859 & 11.6446 & Unk & $1996-2014$ & (Pilegaard et al., 2011) \\
\hline ES-LgS & $\mathrm{OSH}$ & 37.0979 & -2.9658 & Unk & $2007-2009$ & (Reverter et al., 2010) \\
\hline ES-Ln2 & $\mathrm{OSH}$ & 36.9695 & -3.4758 & Unk & 2009-2009 & - \\
\hline FI-Hyy & ENF & 61.8474 & 24.2948 & Unk & $1996-2014$ & (Mammarella et al., 2007) \\
\hline FI-Jok & $\mathrm{CRO}$ & 60.8986 & 23.5135 & Unk & $2000-2003$ & (Lohila, 2004) \\
\hline FI-Lom & WET & 67.9972 & 24.2092 & Unk & $2007-2009$ & - \\
\hline FI-Sod & ENF & 67.3619 & 26.6378 & Unk & $2001-2014$ & (Thum et al., 2007) \\
\hline FR-Fon & DBF & 48.4764 & 2.7801 & $\mathrm{Cfb}$ & $2005-2014$ & (Delpierre et al., 2015) \\
\hline FR-Gri & $\mathrm{CRO}$ & 48.8442 & 1.9519 & $\mathrm{Cfb}$ & $2004-2013$ & (Loubet et al., 2011) \\
\hline FR-LBr & ENF & 44.7171 & -0.7693 & Unk & $1996-2008$ & (Berbigier et al., 2001) \\
\hline FR-Pue & $\mathrm{EBF}$ & 43.7414 & 3.5958 & Unk & $2000-2014$ & (Rambal et al., 2004) \\
\hline IT-BCi & $\mathrm{CRO}$ & 40.5238 & 14.9574 & Unk & 2004-2014 & (Vitale et al., 2015) \\
\hline IT-CA1 & $\mathrm{DBF}$ & 42.3804 & 12.0266 & Unk & $2011-2014$ & (Sabbatini et al., 2016) \\
\hline IT-CA2 & $\mathrm{CRO}$ & 42.3772 & 12.0260 & Unk & 2011-2014 & (Sabbatini et al., 2016) \\
\hline IT-CA3 & $\mathrm{DBF}$ & 42.3800 & 12.0222 & Unk & $2011-2014$ & (Sabbatini et al., 2016) \\
\hline IT-Col & $\mathrm{DBF}$ & 41.8494 & 13.5881 & Unk & 1996-2014 & (Valentini et al., 1996) \\
\hline
\end{tabular}




\begin{tabular}{|c|c|c|c|c|c|c|}
\hline IT-Cp2 & $\mathrm{EBF}$ & 41.7043 & 12.3573 & Unk & 2012-2014 & (Fares et al., 2014) \\
\hline IT-Cpz & $\mathrm{EBF}$ & 41.7052 & 12.3761 & Unk & 1997-2009 & (Garbulsky et al., 2008) \\
\hline IT-Isp & $\mathrm{DBF}$ & 45.8126 & 8.6336 & Unk & 2013-2014 & (Ferréa et al., 2012) \\
\hline IT-La2 & ENF & 45.9542 & 11.2853 & Unk & $2000-2002$ & (Marcolla et al., 2003) \\
\hline IT-Lav & ENF & 45.9562 & 11.2813 & Unk & 2003-2014 & (Marcolla et al., 2003) \\
\hline IT-MBo & GRA & 46.0147 & 11.0458 & Unk & $2003-2013$ & (Marcolla et al., 2011) \\
\hline IT-Noe & $\mathrm{CSH}$ & 40.6061 & 8.1515 & Unk & 2004-2014 & (Papale et al., 2014) \\
\hline IT-PT1 & $\mathrm{DBF}$ & 45.2009 & 9.0610 & Unk & 2002-2004 & (Migliavacca et al., 2009) \\
\hline IT-Ren & ENF & 46.5869 & 11.4337 & Unk & $1998-2013$ & (Montagnani et al., 2009) \\
\hline IT-Ro1 & DBF & 42.4081 & 11.9300 & Unk & $2000-2008$ & (Rey et al., 2002) \\
\hline IT-Ro2 & DBF & 42.3903 & 11.9209 & Unk & 2002-2012 & (Tedeschi et al., 2006) \\
\hline IT-SR2 & ENF & 43.7320 & 10.2910 & Unk & 2013-2014 & - \\
\hline IT-SRo & ENF & 43.7279 & 10.2844 & Unk & 1999-2012 & (Chiesi et al., 2005) \\
\hline IT-Tor & GRA & 45.8444 & 7.5781 & Unk & 2008-2014 & (Galvagno et al., 2013) \\
\hline NL-Hor & GRA & 52.2404 & 5.0713 & Unk & 2004-2011 & (Jacobs et al., 2007) \\
\hline NL-Loo & ENF & 52.1666 & 5.7436 & Unk & $1996-2013$ & (Dolman et al., 2002) \\
\hline RU-Fyo & ENF & 56.4615 & 32.9221 & Unk & $1998-2014$ & (Kurbatova et al., 2008) \\
\hline US-AR1 & GRA & 36.4267 & -99.4200 & Dsa & 2009-2012 & (Raz-Yaseef et al., 2015) \\
\hline US-AR2 & GRA & 36.6358 & -99.5975 & Dsa & 2009-2012 & (Raz-Yaseef et al., 2015) \\
\hline US-ARb & GRA & 35.5497 & -98.0402 & $\mathrm{Cfa}$ & $2005-2006$ & (Raz-Yaseef et al., 2015) \\
\hline US-ARc & GRA & 35.5465 & -98.0400 & $\mathrm{Cfa}$ & $2005-2006$ & (Raz-Yaseef et al., 2015) \\
\hline US-ARM & $\mathrm{CRO}$ & 36.6058 & -97.4888 & Cfa & 2003-2012 & (Fischer et al., 2007) \\
\hline US-Blo & ENF & 38.8953 & -120.6328 & Csa & $1997-2007$ & (Goldstein et al., 2000) \\
\hline US-Cop & GRA & 38.0900 & -109.3900 & Unk & 2001-2007 & (Bowling et al., 2010) \\
\hline US-GBT & ENF & 41.3658 & -106.2397 & Dfc & 1999-2006 & (Zeller and Nikolov, 2000) \\
\hline US-GLE & ENF & 41.3665 & -106.2399 & Dfc & 2004-2014 & (Frank et al., 2014) \\
\hline US-Ha1 & $\mathrm{DBF}$ & 42.5378 & -72.1715 & $\mathrm{Dfb}$ & 1991-2012 & (Urbanski et al., 2007) \\
\hline US-KS2 & $\mathrm{CSH}$ & 28.6086 & -80.6715 & Cwa & 2003-2006 & (Powell et al., 2006) \\
\hline US-Los & WET & 46.0827 & -89.9792 & $\mathrm{Dfb}$ & $2000-2014$ & (Sulman et al., 2009) \\
\hline US-Me1 & ENF & 44.5794 & -121.5000 & $\mathrm{Csb}$ & 2004-2005 & (Irvine et al., 2007) \\
\hline US-Me2 & ENF & 44.4523 & -121.5574 & $\mathrm{Csb}$ & $2002-2014$ & (Irvine et al., 2008) \\
\hline US-Me6 & ENF & 44.3233 & -121.6078 & $\mathrm{Csb}$ & $2010-2014$ & (Ruehr et al., 2012) \\
\hline US-MMS & DBF & 39.3232 & -86.4131 & $\mathrm{Cfa}$ & $1999-2014$ & (Dragoni et al., 2011) \\
\hline US-Myb & WET & 38.0498 & -121.7651 & Csa & 2010-2014 & (Matthes et al., 2014) \\
\hline US-Ne1 & $\mathrm{CRO}$ & 41.1651 & -96.4766 & Dfa & $2001-2013$ & (Verma et al., 2005) \\
\hline US-Ne2 & $\mathrm{CRO}$ & 41.1649 & -96.4701 & Dfa & $2001-2013$ & (Verma et al., 2005) \\
\hline US-Ne3 & $\mathrm{CRO}$ & 41.1797 & -96.4397 & Dfa & $2001-2013$ & (Verma et al., 2005) \\
\hline US-NR1 & ENF & 40.0329 & -105.5464 & Dfc & $1998-2014$ & (Monson et al., 2002) \\
\hline US-ORv & WET & 40.0201 & -83.0183 & $\mathrm{Cfa}$ & 2011-2011 & (Morin et al., 2014) \\
\hline US-PFa & $\mathrm{MF}$ & 45.9459 & -90.2723 & $\mathrm{Dfb}$ & $1995-2014$ & (Desai et al., 2015) \\
\hline US-SRG & GRA & 31.7894 & -110.8277 & Bsk & $2008-2014$ & (Scott et al., 2015) \\
\hline US-SRM & WSA & 31.8214 & -110.8661 & Bsk & 2004-2014 & (Scott et al., 2009) \\
\hline US-Syv & $\mathrm{MF}$ & 46.2420 & -89.3477 & $\mathrm{Dfb}$ & 2001-2014 & (Desai et al., 2005) \\
\hline US-Ton & WSA & 38.4316 & -120.9660 & Csa & 2001-2014 & (Baldocchi et al., 2010) \\
\hline US-Tw1 & WET & 38.1074 & -121.6469 & Csa & 2012-2014 & (Oikawa et al., 2017) \\
\hline US-Tw2 & $\mathrm{CRO}$ & 38.1047 & -121.6433 & Csa & $2012-2013$ & (Knox et al., 2016) \\
\hline
\end{tabular}




\begin{tabular}{lllllll} 
US-Tw3 & CRO & 38.1159 & -121.6467 & $\mathrm{Csa}$ & $2013-2014$ & (Baldocchi et al., 2015) \\
US-Tw4 & WET & 38.1030 & -121.6414 & $\mathrm{Csa}$ & $2013-2014$ & (Baldocchi, 2016) \\
US-Twt & CRO & 38.1087 & -121.6530 & $\mathrm{Csa}$ & $2009-2014$ & (Hatala et al., 2012) \\
US-UMB & DBF & 45.5598 & -84.7138 & $\mathrm{Dfb}$ & $2000-2014$ & (Gough et al., 2013) \\
US-UMd & DBF & 45.5625 & -84.6975 & $\mathrm{Dfb}$ & $2007-2014$ & (Gough et al., 2013) \\
US-Var & GRA & 38.4133 & -120.9507 & $\mathrm{Csa}$ & $2000-2014$ & (Ma et al., 2007) \\
US-WCr & DBF & 45.8059 & -90.0799 & $\mathrm{Dfb}$ & $1999-2014$ & (Cook et al., 2004) \\
US-Whs & OSH & 31.7438 & -110.0522 & $\mathrm{Bsk}$ & $2007-2014$ & (Scott et al., 2015) \\
US-Wi0 & ENF & 46.6188 & -91.0814 & $\mathrm{Dfb}$ & $2002-2002$ & (Noormets et al., 2007) \\
US-Wi3 & DBF & 46.6347 & -91.0987 & $\mathrm{Dfb}$ & $2002-2004$ & (Noormets et al., 2007) \\
US-Wi4 & ENF & 46.7393 & -91.1663 & $\mathrm{Dfb}$ & $2002-2005$ & (Noormets et al., 2007) \\
US-Wi6 & OSH & 46.6249 & -91.2982 & $\mathrm{Dfb}$ & $2002-2003$ & (Noormets et al., 2007) \\
US-Wi9 & ENF & 46.6188 & -91.0814 & $\mathrm{Dfb}$ & $2004-2005$ & (Noormets et al., 2007) \\
US-Wkg & GRA & 31.7365 & -109.9419 & $\mathrm{Bsk}$ & $2004-2014$ & (Scott et al., 2010) \\
\hline
\end{tabular}

$123{ }^{1}$ Plant functional type; see Table 2 for abbreviations.

$124{ }^{2}$ Positive value indicates north latitude.

$125{ }^{3}$ Negative value indicates west longitude.

$126{ }^{4}$ Köppen Climate classification.

1275 “_" indicates that site operators have not provided a reference.

$128{ }^{6}$ Latent and sensible heat flux uncertainty not reported for this site; $50 \%$ uncertainty is 129 assumed. 

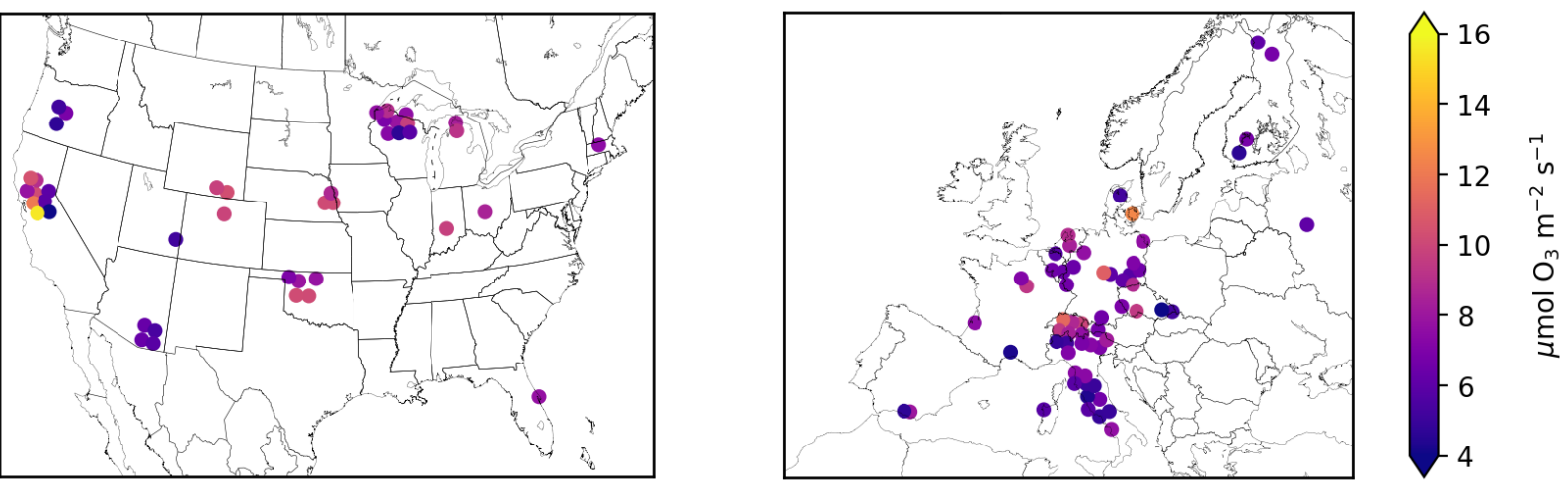

Figure $\mathrm{S} 1$. Mean synthetic total $\mathrm{O}_{3}$ flux $\left(\mathrm{F}_{\mathrm{O}_{3}}^{\mathrm{syn}}\right.$, Sect. 2.1) during the daytime growing season at FLUXNET2015 sites in the United States and Europe. Symbols of some sites have been moved slightly to reduce overlap and improve legibility.

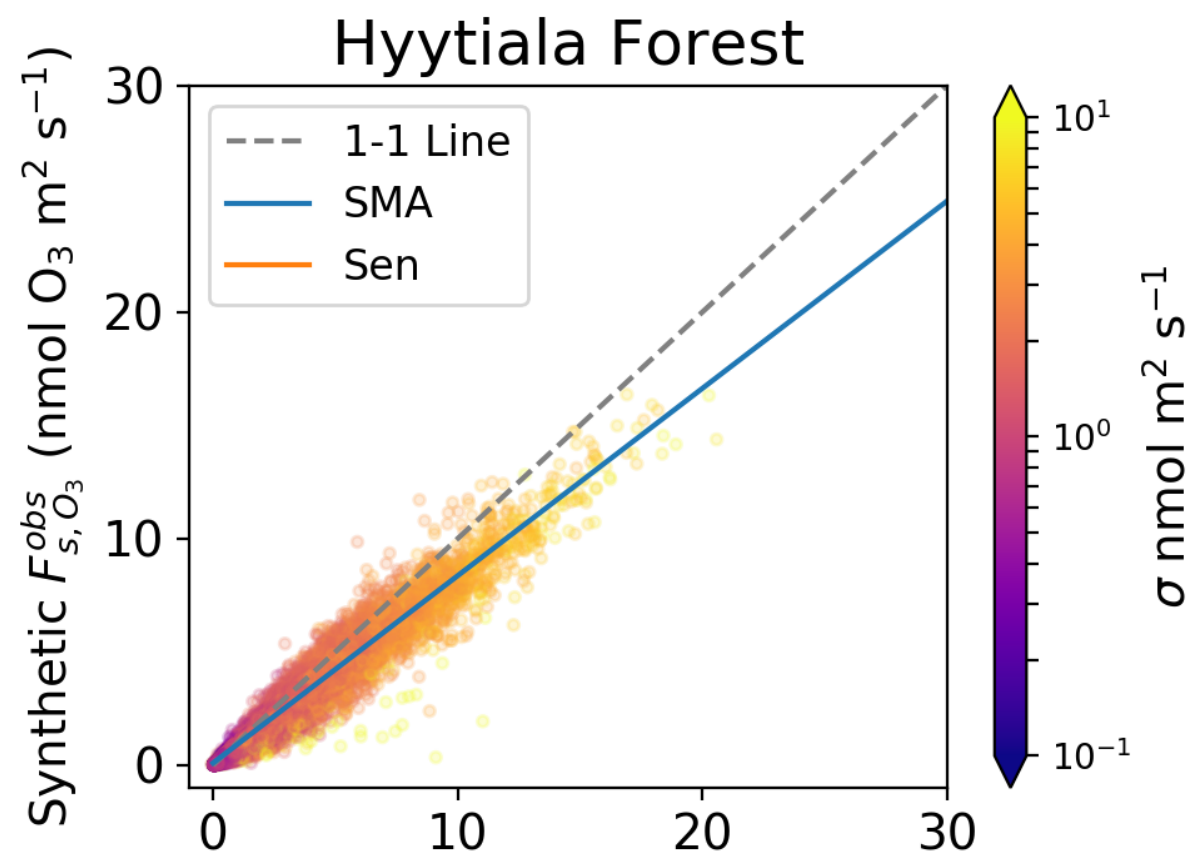

\section{Observation-derived $\mathrm{F}_{\mathrm{s}, \mathrm{O}_{3}}^{\mathrm{obs}}\left(\mathrm{nmol} \mathrm{O}_{3} \mathrm{~m}^{2} \mathrm{~s}^{-1}\right)$}

Figure S2. Synthetic and observed stomatal conductance, $F_{\mathrm{s}, \mathrm{O}_{3}}^{\mathrm{syn}}$, at Hyytiälä Forest illustrating the errors in half-hourly data. Colors show the standard deviation of each value on a logarithmic scale, as calculated by error propagation. 

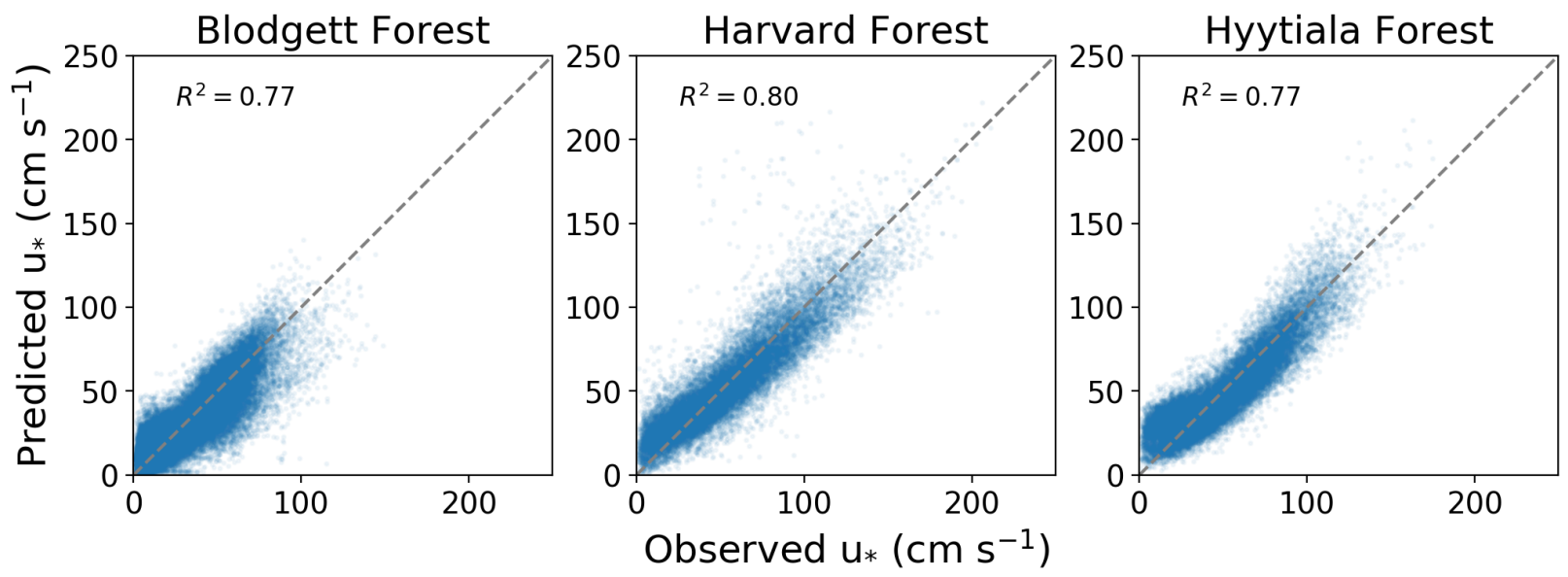

142

Observed $u_{*}\left(\mathrm{~cm} \mathrm{~s}^{-1}\right)$

143 Figure S3. Observed and predicted friction velocity $\left(u_{*}\right)$ from the regression model in Sect 2.3.

144

145

146

147

148

149

150 $\overbrace{i}$

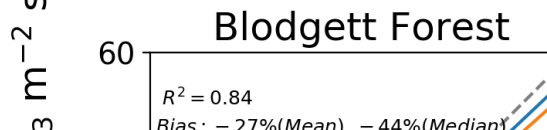

$\mathrm{O}^{m} 40-\begin{aligned} & \text { Bias: }-27 \%(\text { Mean }),-44 \%(\text { Mediany } \\ & \text { slope: } 0.92 \pm 0.01(\text { SMA }), 0.88 \pm 0.01(\text { Sen })\end{aligned}$

$\stackrel{\overline{0}}{\underline{\varepsilon}}$

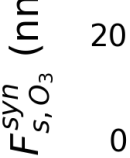

ज
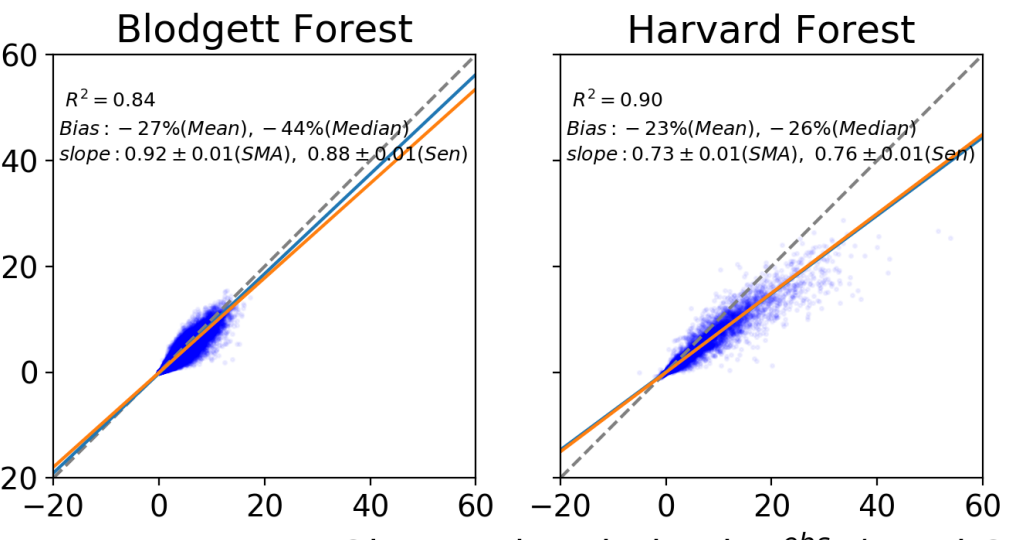

Hyytiala Forest

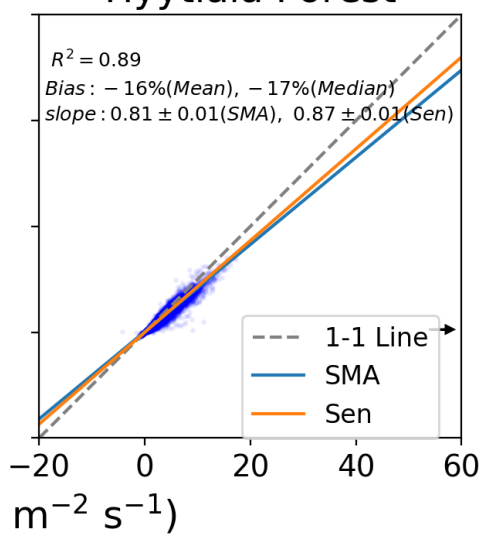

Figure S4. Synthetic and observation-derived half-hourly (hourly at Harvard Forest) stomatal $\mathrm{O}_{3}$ flux. See Fig. 2 for explanation of lines and inset text. 
151

152

153

154

155

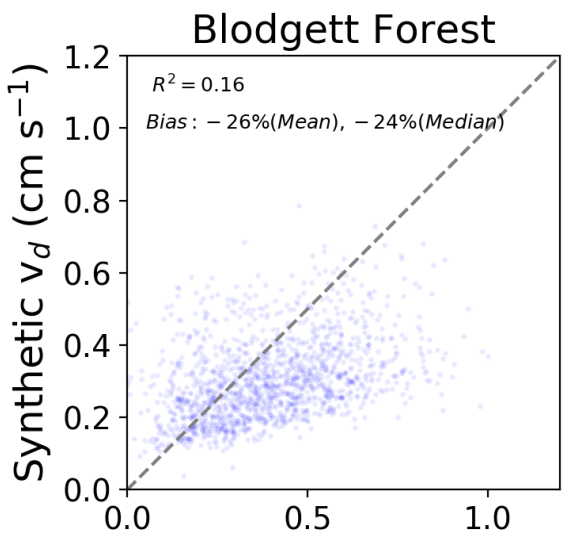

Harvard Forest

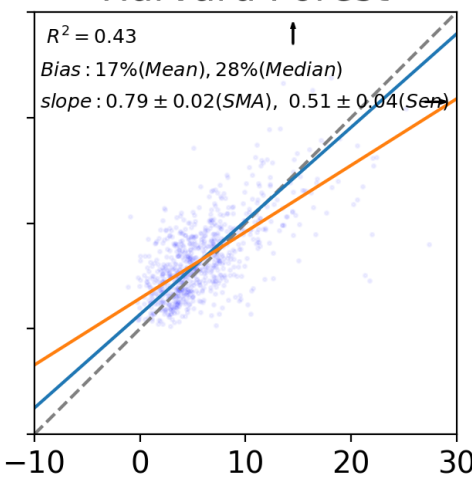

Hyytiala Forest

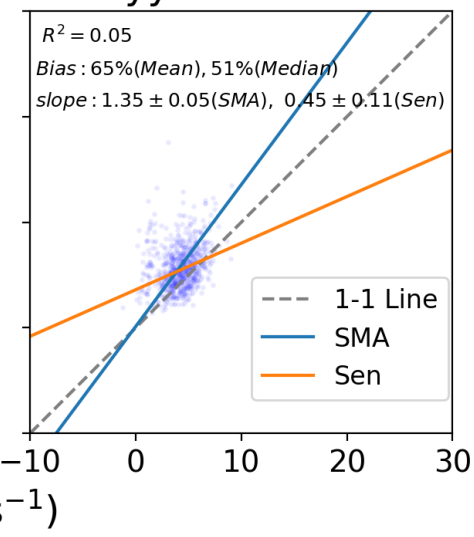

Figure S5. Synthetic and observation-derived daily daytime total $\mathrm{O}_{3}$ flux ( $F_{\mathrm{O}_{3}}^{\mathrm{syn}}$, Sect. 2.1). See Sect. 2.1 for explanation of $F_{\mathrm{O}_{3}}^{\mathrm{syn}}$ and Fig. 2 for explanation of lines and inset text.

156

157 158

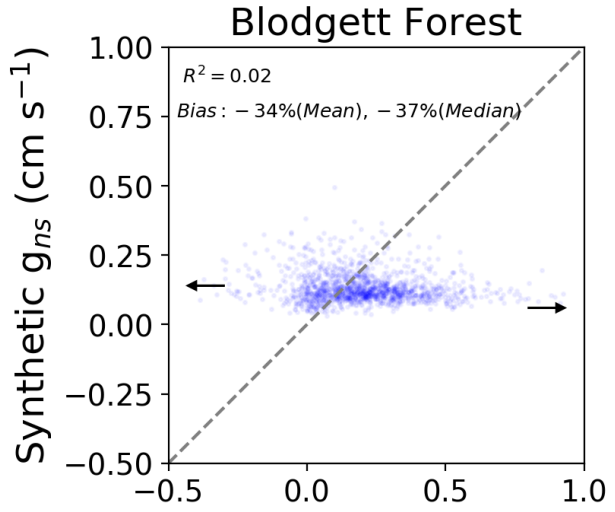

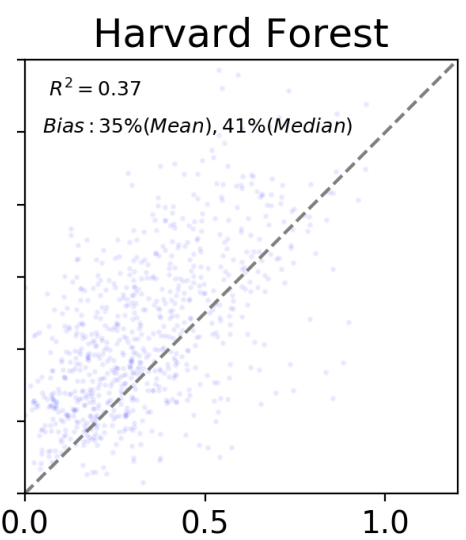

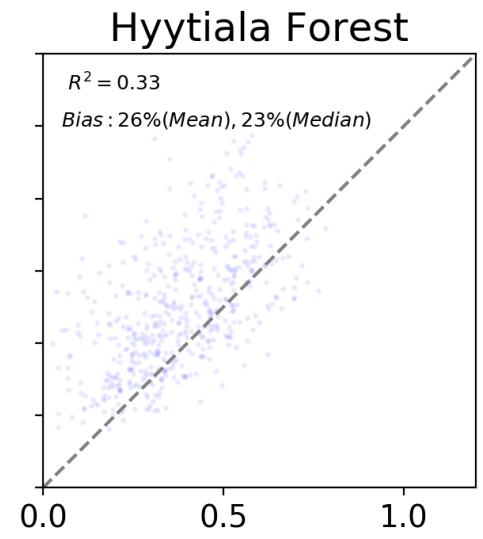

Observation-derived $\mathrm{v}_{d}\left(\mathrm{~cm} \mathrm{~s}^{-1}\right)$

Figure S6. Synthetic and observation-derived daily daytime $\mathrm{O}_{3}$ deposition velocity.
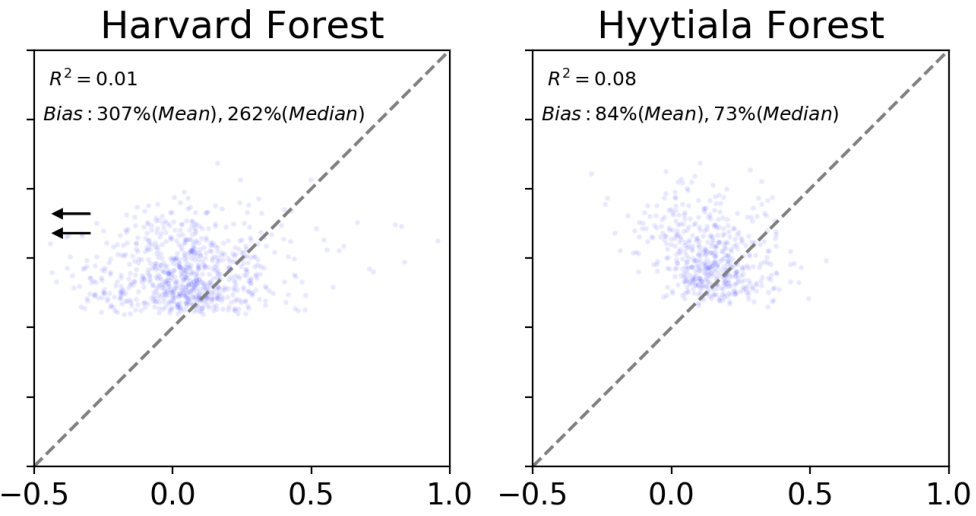

Observation-derived $\mathrm{g}_{n s}\left(\mathrm{~cm} \mathrm{~s}^{-1}\right)$ 
160

161

162

163

164

165

166

167

168

Figure S7. Synthetic and observation-derived daily daytime $\mathrm{O}_{3}$ non-stomatal conductance.
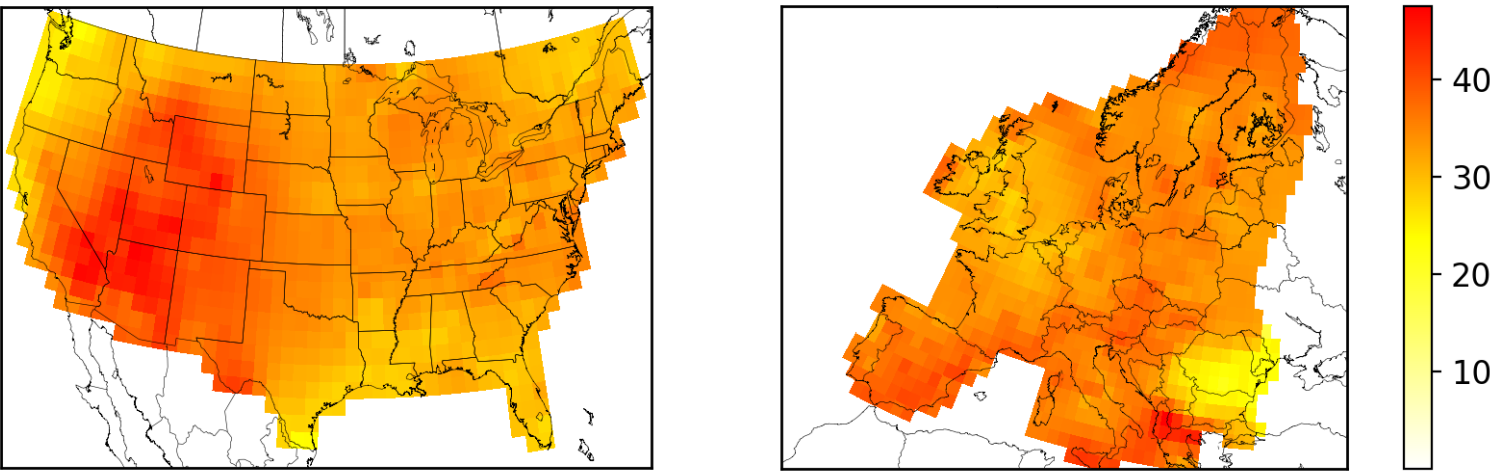

응

Figure S8. Mean daytime (8:00am-8:00pm local) $\mathrm{O}_{3}$ concentrations for the US and Europe during the growing season (typically April-September) for 2000-2014. Data from Schnell et al. (2014). 

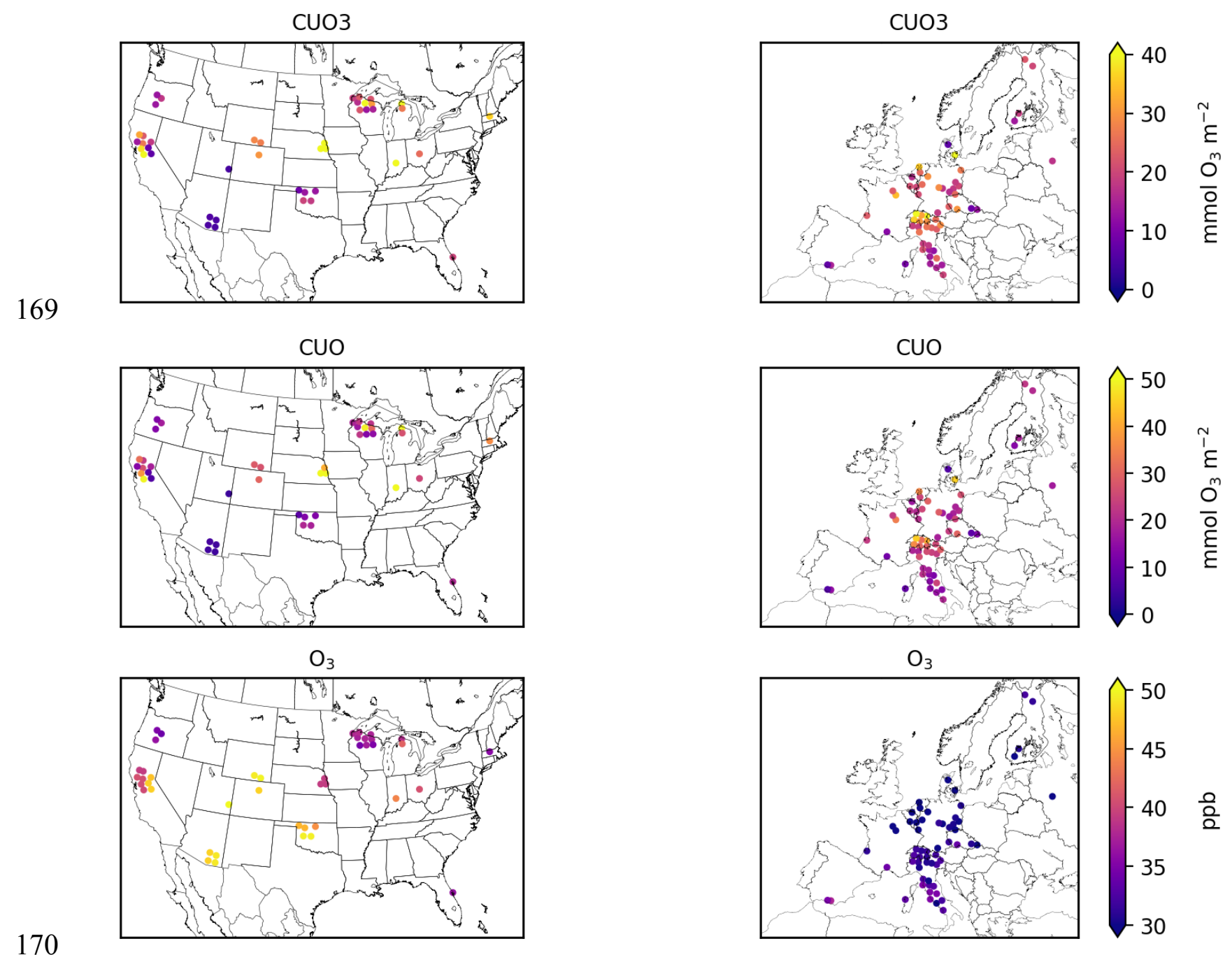

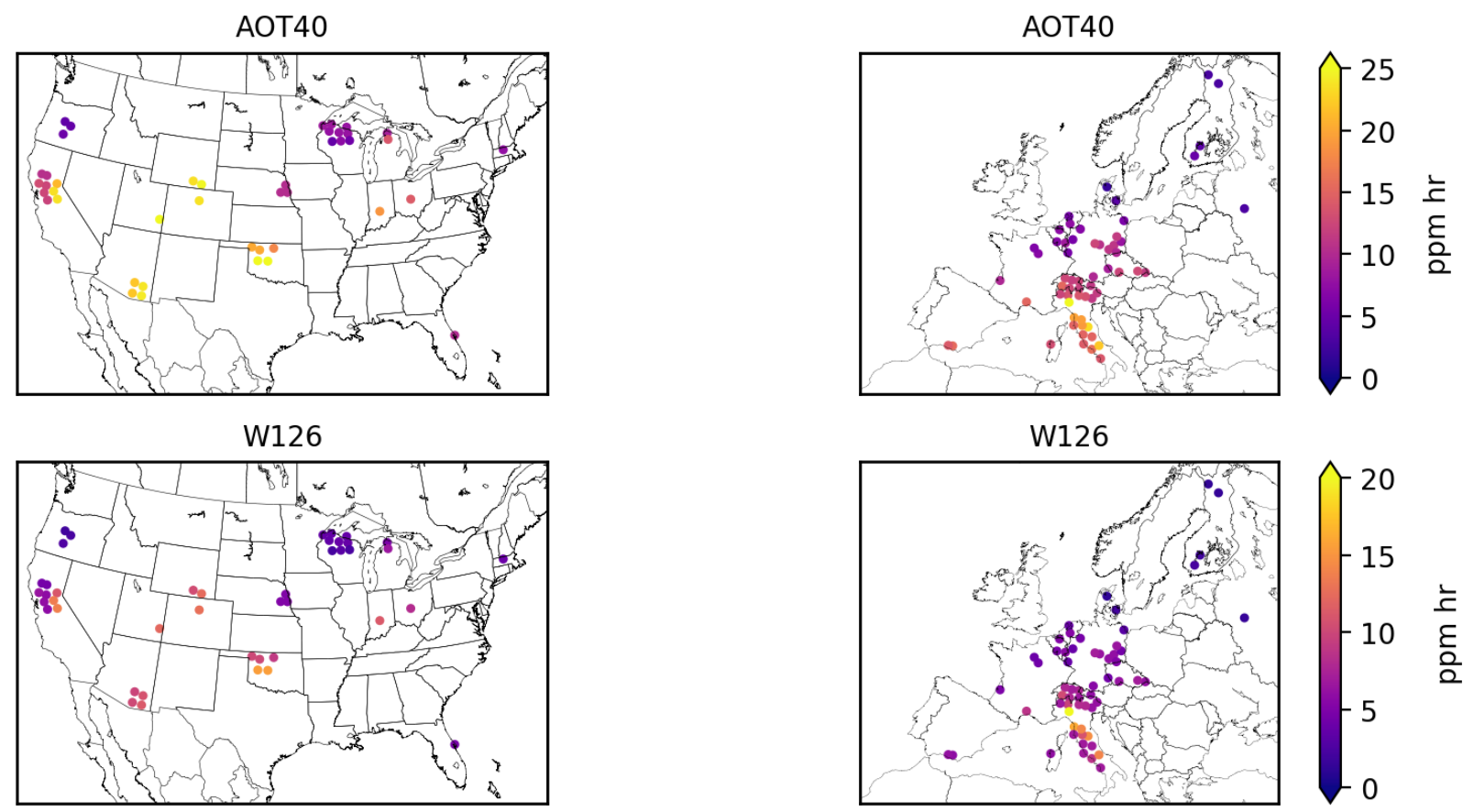

171

Figure S9. Metrics of plant exposure to $\mathrm{O}_{3}$ at FLUXNET2015 sites in the US and Europe: $\mathrm{CUO}_{3}$,

173 CUO, mean $\mathrm{O}_{3}$, AOT40, and W126. See Sect. 3.4 for metric definitions.

174

175 